\title{
Large aperture vibrating wire monitor with two mechanically coupled wires for beam halo measurements
}

\author{
S. G. Arutunian, * A. E. Avetisyan, M. M. Davtyan, G. S. Harutyunyan, and I. E. Vasiniuk \\ Yerevan Physics Institute, Alikhanian Brothers Street 2, 375036 Yerevan, Armenia \\ M. Chung, ${ }^{\dagger}$ and V. Scarpine \\ Fermi National Accelerator Laboratory, Batavia, Illinois 60510, USA
}

(Received 14 April 2013; published 19 March 2014)

\begin{abstract}
Development of a new type of vibrating wire monitor (VWM), which has two mechanically coupled wires (vibrating and target), is presented. The new monitor has a much larger aperture size than the previous model of the VWM, and thus allows us to measure transverse beam halos more effectively. A prototype of such a large aperture VWM with a target wire length of $60 \mathrm{~mm}$ was designed, manufactured, and bench-tested. Initial beam measurements have been performed at the Fermilab High Intensity Neutrino Source facility, and key results are presented.
\end{abstract}

DOI: 10.1103/PhysRevSTAB.17.032802

PACS numbers: 29.27.Fh

\section{INTRODUCTION}

The precise measurement and control of transverse beam halos are critical for achieving the performance goals of many high-intensity linear and circular accelerators. To characterize the beam halo, it is desirable to measure the beam core and halo simultaneously. Therefore, methods with very high dynamic range are often required [1,2]. Over the past decade, significant advances have been made in the field of halo diagnostics. For example, a novel beam halo imaging technique with a digital optical mask has recently been developed [3,4]. More detailed overviews of different methods are presented in Refs. [1,2].

For nondestructive transverse beam profile and halo measurements, wire scanners are still widely used. Recently, a new class of wire scanner, the so-called vibrating wire monitor (VWM) [5-9] was developed to improve the dynamic range of conventional wire scanners and also to reduce the size of the measurement system. The VWM system is relatively compact, so a large quantity of VWM's can be installed in a beam line.

The operating principle of the VWM is based on the measurement of the change in the natural oscillation frequency (i.e., characteristic frequency or normal frequency) of a vibrating wire. The mode of natural oscillation in the wire is generated by the interaction of an ac drive current through the wire with a permanent magnetic field. Note that the vibrating wire is connected to a positive

\footnotetext{
s_arut@yahoo.com

†mchung@fnal.gov
}

Published by the American Physical Society under the terms of the Creative Commons Attribution 3.0 License. Further distribution of this work must maintain attribution to the author $(s)$ and the published article's title, journal citation, and DOI. feedback circuit. The circuit consists of operational amplifiers (LM833) with a resistance on order of a few hundred $\mathrm{k} \Omega$, and amplifies oscillation at a certain natural frequency (the second harmonic in this work). The amplitude of the drive current is stabilized by means of a voltage-controlled resistor in a negative feedback loop on the level of a few $\mathrm{mA}$. The vibrating wire is directly connected to the feedback circuit by wires of length 1-2 $\mathrm{m}$, so the circuit can be placed outside the vacuum chamber. The output signal from the feedback circuit is amplified and converted into a square waveform. The square wave signal can be transferred over long distances (a few hundred meters) and then used for frequency counting. Some other details of VWM operation principle are described in Refs. [8,9].

A frequency shift arises when beam particles penetrate the wire and change the wire temperature. For charged particles, ionization loss is the main contributor to the energy transfer (see, for example, the power deposition formula (1) of Ref. [6]).

To keep the central part of the VWM open for the beam to pass, excitation of the second harmonic of the wire oscillations is normally considered. In this case, $\sim 2 / 3$ of the wire should be immersed in the magnetic field and only $\sim 1 / 3$ of the wire is available as a target for the beam measurements. The schematic of the original design of the VWM with a $36 \mathrm{~mm}$ wire length is presented in Fig. 1. Four $\mathrm{SaCo}$ (samarium cobalt) magnets with a $9 \mathrm{~mm}$ diameter provide dipole magnetic fields (of opposite polarity) in the vicinity of the wire ends. The magnetic field strength is about $0.5-0.7 \mathrm{~T}$. The dynamic range of such a VWM was about $10^{6}$, and it was possible to measure a 6 sigma range of the proton beam with a transverse size of about 5-6 mm $[10,11]$. However, the aperture size of the VWM was only $\sim 12 \mathrm{~mm}$, so it was noticed that indeed a lot of halo particles were deposited on the structural components of the 


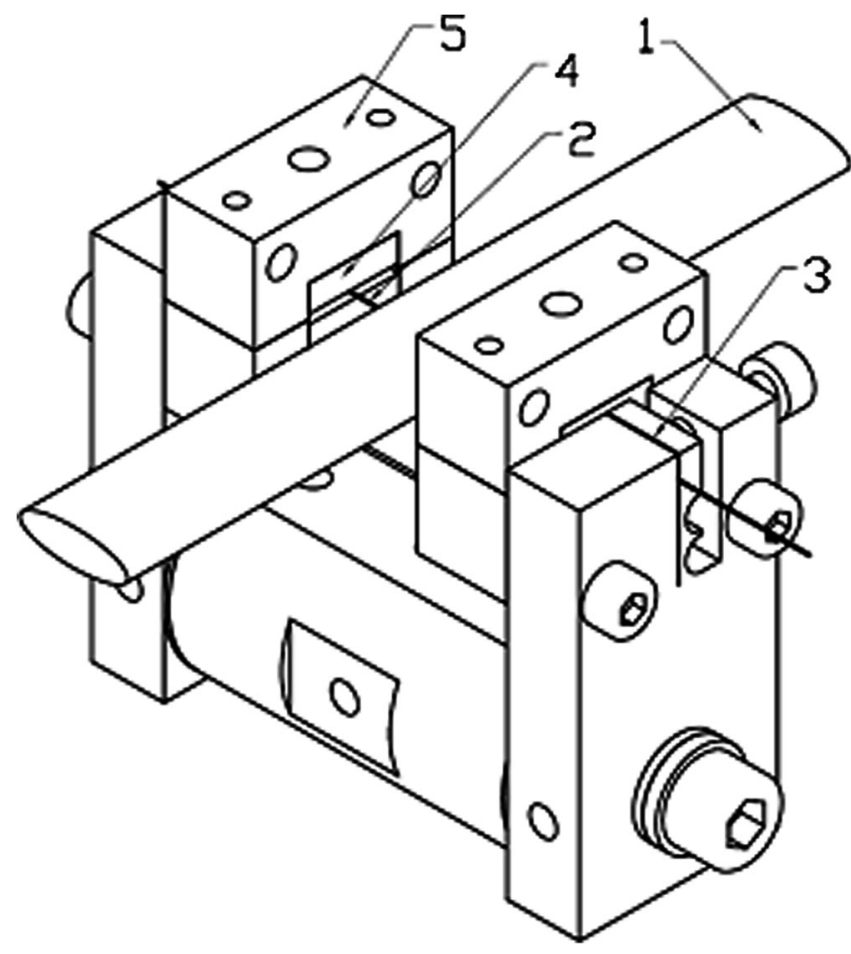

FIG. 1. The main view of the original design of the VWM. 1: beam; 2: vibrating wire; 3 : clips; 4: magnet; 5 : magnet holder. Here, the vibrating wire serves as a target for the beam at the same time. The wire length is $36 \mathrm{~mm}$, but only $\sim 1 / 3$ of the wire is available as a target for the beam measurements.

monitor, making the interpretation of the experimental results very difficult [11]. A simple way to avoid this situation would be to extend the length of the wire and to provide the required aperture size. In this case, however, the size of the whole VWM system has to increase and also the response time of the sensor becomes slower.

In this paper we solve this problem by developing a new type of VWM that consists of two mechanically coupled wires. One of the wires remains as a vibrating wire, and measures the strain of the other wire (target wire). Only the target wire is exposed to the beam. Since the target wire is not in the magnet, the aperture size of the proposed monitor can be made much larger than the previous design without increasing the overall monitor size. In addition, the target wire is not connected to the feedback circuit, and thus can be made of nonconductive materials (e.g., dielectrics). Use of materials with extraordinarily high thermal conductivity can lead to a decrease in the monitor's response time, which is very desirable in some applications.

A prototype of such a Large aperture vibrating wire monitor (LA-VWM) with a target wire length of $60 \mathrm{~mm}$ was developed and manufactured. In this work, we present the bench test results of the LA-VWM. Dependencies of the LA-VWM signals on the target and environmental temperatures were estimated analytically, the response time of the LA-VWM was investigated by illuminating the target wire by laser, and the response of the LA-VWM to the target wire temperature change was characterized by applying dc currents to the wire.

There are plans to use a LA-VWM for the investigation of the beam halo at the Project-X Injector Experiment (PXIE), which is a research and development program to address accelerator physics and technology issues for the planned multi-MW superconducting proton accelerator facility $[12,13]$. Initial measurements have been performed at the Fermilab High Intensity Neutrino Source (HINS) facility [13-15], and key results are presented in Sec. VI of this paper.

\section{DESCRIPTION OF THE VIBRATING WIRE MONITOR}

In the original design of the VWM, we used the vibrating wire as a direct target for the particle beam. The requirement to immerse some portion of the vibrating wire in a magnetic field for oscillation generation reduced the effective aperture size of the VWM. Because an ac current needed to flow through the wire for oscillation generation, the wire material must have been conductive, which prevented us from using a variety of dielectric materials with good thermal properties. One idea to avoid such restrictions was to use two mechanically coupled wires, in which one serves as a vibrating wire and the other as a sensitive target. This idea was originally suggested in Ref. [11] and was realized in Ref. [16]. In this modified configuration, the target and vibrating wires were coupled by a balanced arm with a bearing axis, and the two wires were aligned along a straight line. Unfortunately, this type of VWM substantially increased the dimensions of the VWM and made the installation inside a vacuum chamber very difficult. For example in Ref. [16], the lengths of the target and vibrating wires were 50 and $36 \mathrm{~mm}$, respectively, and the transverse dimension of the VWM became $130 \times 145 \mathrm{~mm}$.

The new modification of the VWM proposed in this work can solve all of the above-mentioned problems, i.e., it provides large aperture size, keeps the VWM compact, and allows the use of a wide range of materials both for vibrating and target wires. The new idea is to couple the two wires with a balancing arm and install the wires on opposite ends of the arm. A schematic view of this concept is presented in Fig. 2.

We suppose that both wires are under tension, i.e., at least one of the wires (when it is loose) is shorter than the gap between the two parallel arms (unmovable and balancing arms shown in Fig. 2). The frequency of the second harmonics of the vibrating wire is given by

$$
F=\frac{1}{l_{1}^{0}} \sqrt{\frac{\varepsilon_{1} E_{1}}{\rho_{1}}},
$$




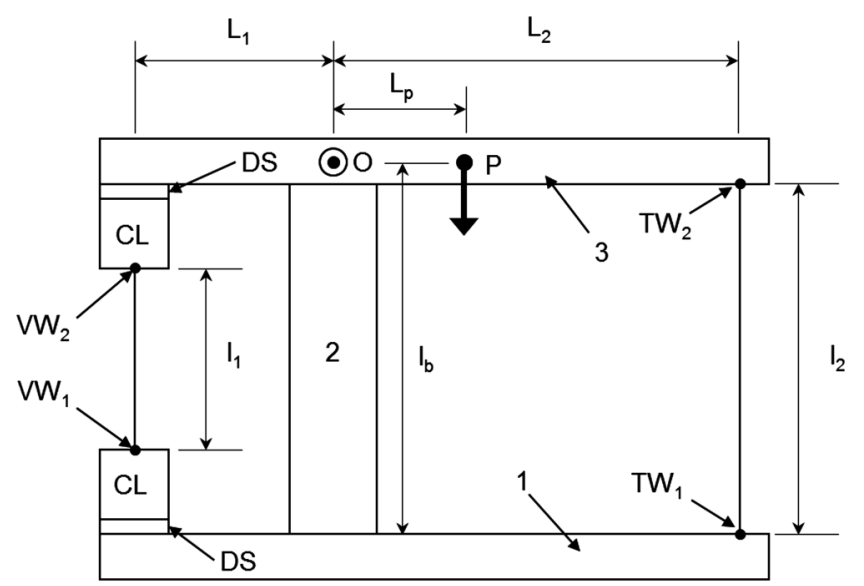

FIG. 2. Simplified drawing of the LA-VWM. 1: unmovable arm; 2: balancing arm support; 3: balancing arm. The thick arrow at point $\mathrm{P}$ denotes the direction of weight of the balancing arm applied to its center of gravity. The following notations are used in the text: $l_{1}$ : length of vibrating wire (distance between points $\mathrm{VW}_{1}$ and $\mathrm{VW}_{2}$ ); $l_{2}$ : length of target wire (distance between points $\mathrm{TW}_{2}$ and $\left.\mathrm{TW}_{2}\right) ; l_{b}$ : length of balancing arm support (vertical distance between point $\mathrm{TW}_{1}$ and balancing arm axis $\mathrm{O}$ ); $L_{1}$ : horizontal distance between vibrating wire and point $\mathrm{O}$; $L_{2}$ : horizontal distance between target wire and point $\mathrm{O} ; L_{P}$ : horizontal distance between center of gravity of balancing arm (point $\mathrm{P}$ ) and arm axis (point $\mathrm{O}$ ), $l_{k}$-vertical distance between points $\mathrm{TW}_{2}$ and $\mathrm{O} ; l_{p}$ : total thickness of dielectric spacers (DS); $l_{z}$ : total length of vibrating wire clips CL. The target wire length $l_{2}$ is $60 \mathrm{~mm}$, and $100 \%$ of the wire is available as a target for the beam measurements.

where $\rho_{1}$ and $E_{1}$ are the density and elasticity modulus of the vibrating wire material, $\varepsilon_{1}=\left(l_{1}-l_{1}^{0}\right) / l_{1}^{0}$ is tensile strain, and $l_{1}\left(l_{1}^{0}\right)$ is the length of the strained (unstrained) vibrating wire. We also assume that all the VWM parts are rigid compared to the wire. Therefore, the parameter $l_{1}$ can be defined by the distance between two clips (denoted by $\mathrm{CL}$ in Fig. 2). Because of the coupling between the vibrating and target wires, we have the following equations for moments and torque balance around point $\mathrm{O}$ (Fig. 2) for the whole system:

$$
\begin{aligned}
l_{k}+l_{p}+l_{z}+l_{1}+L_{1} \theta & =l_{b}, \quad l_{k}+l_{2}-L_{2} \theta=l_{b}, \\
L_{1} S_{1} E_{1} \frac{l_{1}-l_{1}^{0}}{l_{1}^{0}}-P L_{p} & =L_{2} S_{2} E_{2} \frac{l_{2}-l_{2}^{0}}{l_{2}^{0}}
\end{aligned}
$$

where $l_{2}\left(l_{2}^{0}\right)$ is the length of the strained (unstrained) target wire, $\theta$ is the angle between the unmovable and balancing arms (assumed small), $S_{1}\left(S_{2}\right)$ is the cross section of the vibrating (target) wire, $E_{2}$ is the elasticity modulus of the target wire material, and $P$ is the weight of the balancing arm. For other notations, see the caption of Fig. 2. The first two equations in Eq. (2) are derived from the geometrical configuration of the wire coupling, and the last equation represents the moments/torque balance around point $\mathrm{O}$. By solving the simultaneous equations (2) for $l_{1}, l_{2}$, and $\theta$, we found the following expression for $\varepsilon_{1}$ :

$$
\varepsilon_{1}=\frac{L_{2} S_{2} E_{2}\left(L_{1} \delta_{2} l_{2}^{0}+L_{2} \delta_{1} l_{1}^{0}\right)+L_{1} l_{2}^{0} P L_{p}}{L_{1}^{2} S_{1} E_{1} l_{2}^{0}+L_{2}^{2} S_{2} E_{2} l_{1}^{0}}
$$

where

$$
\delta_{1}=\frac{l_{b}-l_{p}-l_{k}-l_{z}-l_{1}^{0}}{l_{1}^{0}}, \quad \delta_{2}=\frac{l_{b}-l_{k}-l_{2}^{0}}{l_{2}^{0}} .
$$

Here, we assumed that the balancing arm is nearly in parallel with the unmovable arm when the wires are under tension and neglect the higher order terms for small values $\delta_{1}$ and $\delta_{2}$.

Temperature change in the target wire leads to a change of $l_{2}^{0}$ according to

$$
l_{2}^{0} \rightarrow l_{2}^{0}\left(1+\alpha_{2} \Delta T\right)
$$

where $\alpha_{2}$ is the thermal expansion coefficient of the target wire, and $\Delta T$ is the temperature change relative to the initial temperature. Note that all the other parts of the VWM are assumed to have the same initial temperature. In this case, we have $\delta_{2}=\delta_{2}^{0}-\alpha_{2} \Delta T$ from Eq. (4) and $\varepsilon_{1}=$ $\varepsilon_{1}^{0}-\alpha_{2} A \Delta T$ from Eq. (3), where $\delta_{2}^{0}$ and $\varepsilon_{1}^{0}$ represent the values at the initial temperature $(\Delta T=0)$. The dimensionless geometrical parameter $A$ is defined as

$$
A=\frac{L_{1} L_{2} S_{2} E_{2} l_{2}^{0}}{L_{1}^{2} S_{1} E_{1} l_{2}^{0}+L_{2}^{2} S_{2} E_{2} l_{1}^{0}} .
$$

Inserting the expression $\varepsilon_{1}=\varepsilon_{1}^{0}-\alpha_{2} A \Delta T=\varepsilon_{1}^{0}(1-$ $\left.\alpha_{2} A \Delta T / \varepsilon_{1}^{0}\right)$ into Eq. (1) with the definition of $A$ in Eq. (6), we have

$$
F=\frac{1}{l_{1}^{0}} \sqrt{\frac{E_{1} \varepsilon_{1}^{0}\left(1-\alpha_{2} A \Delta T / \varepsilon_{1}^{0}\right)}{\rho_{1}} .}
$$

After expanding in powers of the small quantity, $\alpha_{2} A \Delta T / \varepsilon_{1}^{0}$, and neglecting higher order terms, we finally obtain the dependence of the vibrating wire frequency change on the target wire temperature change as

$$
\frac{\Delta F}{\Delta T}=-\frac{\alpha_{2} E_{1}}{2 F_{0}\left(l_{1}^{0}\right)^{2} \rho_{1}} A,
$$

where $F_{0}=\left(1 / l_{1}^{0}\right) \sqrt{\varepsilon_{1}^{0} E_{1} / \rho_{1}}$ is the initial frequency of the vibrating wire and $\Delta F=F-F_{0}$. It is interesting to compare this expression to the case of a one-wire sensor [9], where the vibrating wire serves as a target wire at the same time: 


$$
\frac{\Delta F}{\Delta T}=-\frac{\alpha_{1} E_{1}}{2 F_{0}\left(l_{1}^{0}\right)^{2} \rho_{1}}
$$

This formula differs from Eq. (8) by a factor $A$, which is about unity. Also we note that in Eq. (9), the thermal expansion coefficient of the vibrating wire $\alpha_{1}$ is used instead of the target wire, $\alpha_{2}$.
In the case of ambient temperature change, we should take into account the thermal expansions of all the parts of the monitor. Similar to Eq. (5), we consider the length change of each part in the form of $l \rightarrow l(1+\alpha \Delta T)$. After neglecting the higher order terms, we obtain the following expressions from Eq. (4):

$$
\delta_{1}=\delta_{1}^{0}-\Delta T \frac{l_{b}\left(\alpha_{b}-\alpha_{1}\right)-l_{p}\left(\alpha_{p}-\alpha_{1}\right)-l_{k}\left(\alpha_{k}-\alpha_{1}\right)-l_{z}\left(\alpha_{z}-\alpha_{1}\right)}{l_{1}^{0}}, \quad \delta_{2}=\delta_{2}^{0}-\Delta T \frac{l_{b}\left(\alpha_{b}-\alpha_{2}\right)-l_{k}\left(\alpha_{k}-\alpha_{2}\right)}{l_{2}^{0}},
$$

where each subscript of the thermal expansion coefficient $\alpha$ indicates the corresponding part of the monitor. After inserting Eq. (3) into Eq. (1) with $\delta_{1}$ and $\delta_{2}$ defined in Eq. (10), and expanding in powers of the small quantities, we obtain the following approximate expression for the dependence of the vibrating wire frequency on the ambient temperature:

$$
\frac{\Delta F}{\Delta T_{\mathrm{AMBIENT}}}=-\frac{E_{1}}{2 F_{0}\left(l_{1}^{0}\right)^{2} \rho_{1}} B
$$

where

$$
B=\frac{L_{2} S_{2} E_{2}\left\{L_{2}\left[l_{b}\left(\alpha_{b}-\alpha_{1}\right)-l_{p}\left(\alpha_{p}-\alpha_{1}\right)-l_{k}\left(\alpha_{k}-\alpha_{1}\right)-l_{z}\left(\alpha_{z}-\alpha_{1}\right)\right]+L_{1}\left[l_{b}\left(\alpha_{b}-\alpha_{2}\right)-l_{k}\left(\alpha_{k}-\alpha_{2}\right)\right]\right\}}{L_{1}^{2} S_{1} E_{1} l_{2}^{0}+L_{2}^{2} S_{2} E_{2} l_{1}^{0}} .
$$

\section{LA-VWM ASSEMBLY}

A prototype of the LA-VWM with an aperture size of $60 \mathrm{~mm}$ was designed and manufactured. The main balancing arm and the unmovable arm were made of stainless steel and connected to each other through a supporting rod made of Invar. The vibrating wire was made of stainless steel with a length of $36 \mathrm{~mm}$ and placed inside the magnet system. The magnet system provided two magnetic dipole configurations with opposite polarities, and was

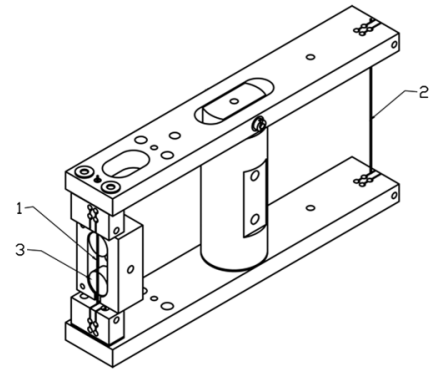

(a)

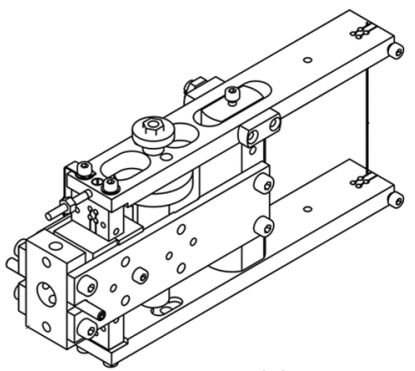

(b)
FIG. 3. (a) Main parts of the LA-VWM: 1: vibrating wire; 2: target wire; 3: round magnets providing the magnetic field for wire oscillation generation. (b) Assembled view of the LAVWM. The space to the right of the balancing arm support is free for beam measurement (in comparison with the previous model of the VWM in Fig. 1). constructed with $\mathrm{SaCo}$ permanent magnets. The axis of the balancing arm was equipped with miniature bearings. For the target wire, tungsten was chosen to decrease the response time of the monitor for the bench test (see below for more details). For electrical insulation, dielectric spacers $(0.1 \mathrm{~mm}$ thick mylar) were inserted. In Fig. 3(a), the main parts of the LA-VWM are shown. In Fig. 3(b), the assembled view of the LA-VWM is presented.

The dependence of the vibrating wire frequency on the target wire temperature is estimated according to Eq. (8)

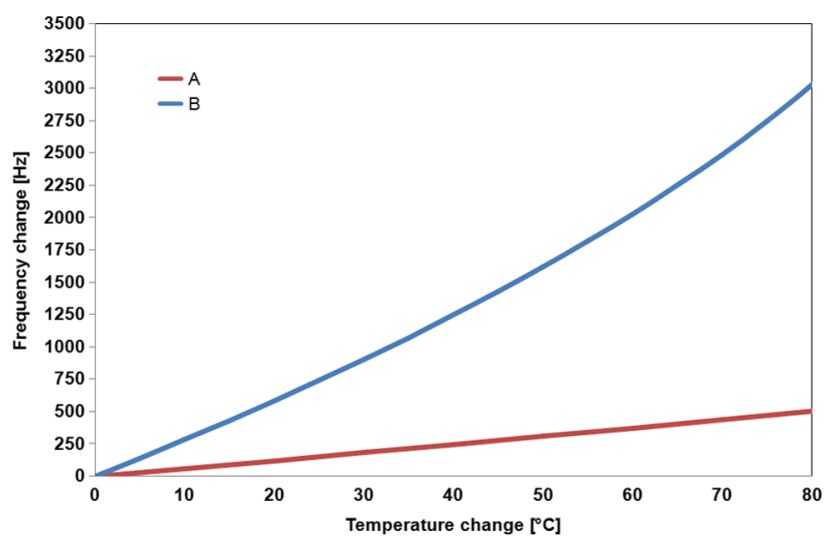

FIG. 4. Frequency dependence on the target wire temperature (curve A). Frequency dependence on the ambient temperature (curve B). 
assuming that all the other parts of the VWM remain at the initial temperature, and is presented in Fig. 4 (curve A). For small temperature changes, the slope of the dependence is about $6 \mathrm{~Hz} / \mathrm{K}$. The dependence of the vibrating wire frequency on the ambient temperature is also estimated according to Eq. (11) and shown in Fig. 4 (curve B). The slope of the linear dependence for small ambient temperature changes is about $28.5 \mathrm{~Hz} / \mathrm{K}$.

The difference in slope between Fig. 4 curve A and curve $\mathrm{B}$ is mainly due to the low thermal expansion coefficient of the tungsten target wire. As mentioned above, the target material choice for the bench test was based on the response time not the sensitivity. We also note that the target wire temperature will rise much higher than the ambient temperature.

\section{LA-VWM RESPONSE TIME}

One of the important parameters of the VWM is its response time. This parameter depends on the thermal conductivity properties of the target wire material, the wire length, and the environment in which the monitor is placed. In air, in addition to the thermal conduction through the wire, convective heat transport should be considered as well. At room and moderately high temperatures, the radiation losses are negligible.

If some power is deposited on the target wire, its temperature will increase until the incoming power is balanced by the heat sink through all the relevant mechanisms. The time required to reach $62.3 \%$ (i.e., $1-\mathrm{e}^{-1}$ ) of the new equilibrium temperature is called the device's response time (or time constant) [17]. To estimate the response time of the LA-VWM in vacuum, we used a simple model of a triangular distribution of the wire temperature. The temperature increase is maximum at the wire center, decreases linearly, and eventually becomes zero at both ends of the wire (see Fig. 5). We denote the maximum temperature increase with respect to the ambient temperature by $\Delta T$.

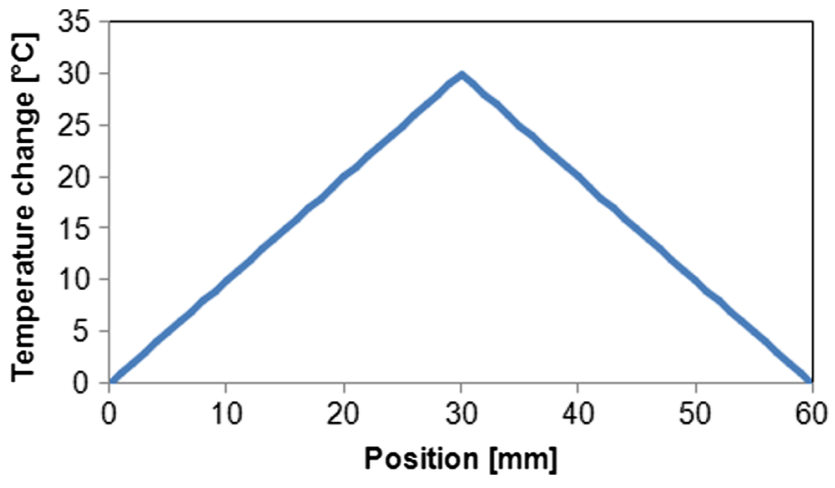

FIG. 5. Triangular distribution of the target wire temperature change in LA-VWM.
If the wire is placed in a vacuum, the only heat sink mechanism is the thermal conduction along the wire, and the power needed to support this temperature distribution is equal to

$$
W=\frac{4 \lambda S}{l} \Delta T
$$

where $l$ is the target wire's length, $\lambda$ is the wire material's thermal conductivity coefficient, and $S$ is the wire's crosssectional area. In Eq. (12) we assume that there are two equal heat sinks (from the wire center to the wire ends). The required response time $\tau$ can be found from the following thermal equation

$$
W \tau=S l \rho c \Delta T / 2,
$$

where $\rho$ is the density and $c$ is the specific heat coefficient of the target wire. Equation (13) is a simple relation that allows us to estimate how much time $\tau$ is needed to increase the temperature of the wire with mass $S l \rho$ and mean temperature $\Delta T / 2$. In our previous work (see Ref. [5]), we have shown that these simple estimations are in reasonably good agreement (within an order of magnitude) with the exact solutions based on heat transfer partial differential equations. From Eqs. (12) and (13), we find

$$
\tau=\frac{\rho c l^{2}}{8 \lambda} .
$$

In Table I, the values of $\tau$ for different materials are presented (the wire length $l$ is set to $61.2 \mathrm{~mm}$ for all materials). We include the exotic material graphen, which has an extraordinarily high thermal conductivity [18]. Graphen-based materials are available on the market due to the groundbreaking nano-materials synthesis technology that produces graphen from carbon dioxide $[19,20]$. As we can expect from Table I, the use of graphen even as a coating material of the target wire could considerably reduce the detector's response time. For example, coating

TABLE I. Response time for different target wire materials.

\begin{tabular}{lrccc}
\hline \hline Material & $\tau[\mathrm{sec}]$ & $\rho\left[\mathrm{g} / \mathrm{cm}^{3}\right]$ & $c[\mathrm{~J} /(\mathrm{g} * \mathrm{~K})]$ & $\lambda[\mathrm{W} / \mathrm{cm} * \mathrm{~K}]$ \\
\hline Gold & 3.67 & 19.3 & 0.129 & 3.18 \\
\hline Silver & 2.72 & 10.5 & 0.237 & 4.29 \\
\hline Platinum & 18.65 & 21.45 & 0.133 & 0.716 \\
\hline Tungsten & 6.95 & 19.3 & 0.133 & 1.73 \\
\hline Invar & 145.50 & 8 & 0.505 & 0.13 \\
\hline $\begin{array}{l}\text { Stainless } \\
\text { Steel }\end{array}$ & 114.77 & 7.96 & 0.502 & 0.163 \\
\hline Graphen & 0.38 & 2.23 & 0.72 & 20 \\
\hline \hline
\end{tabular}




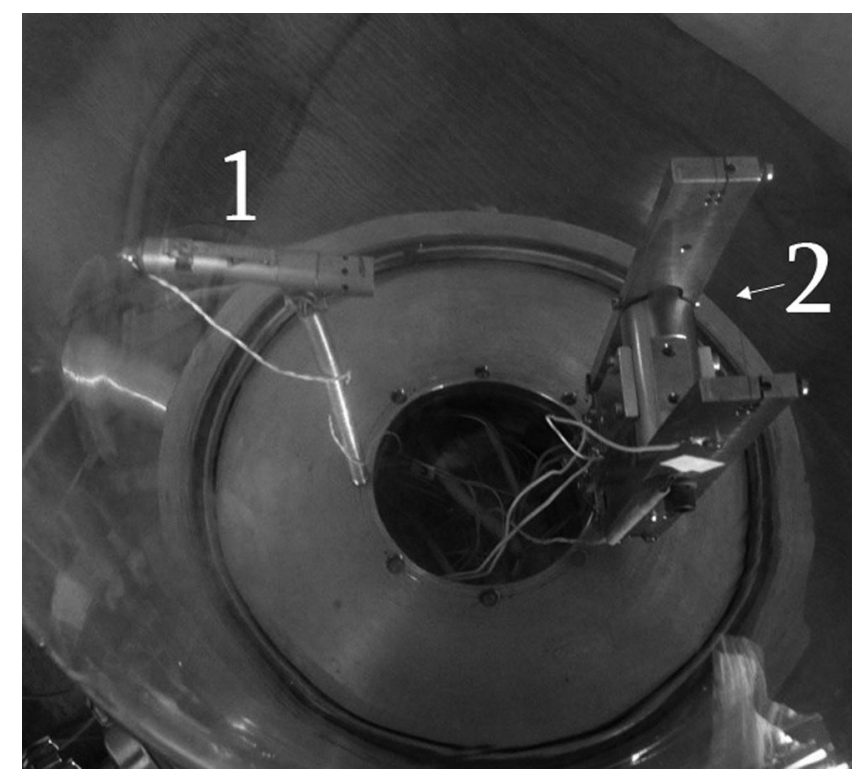

FIG. 6. Experimental setup for response time measurement. 1: diode laser; 2: VWM target wire.

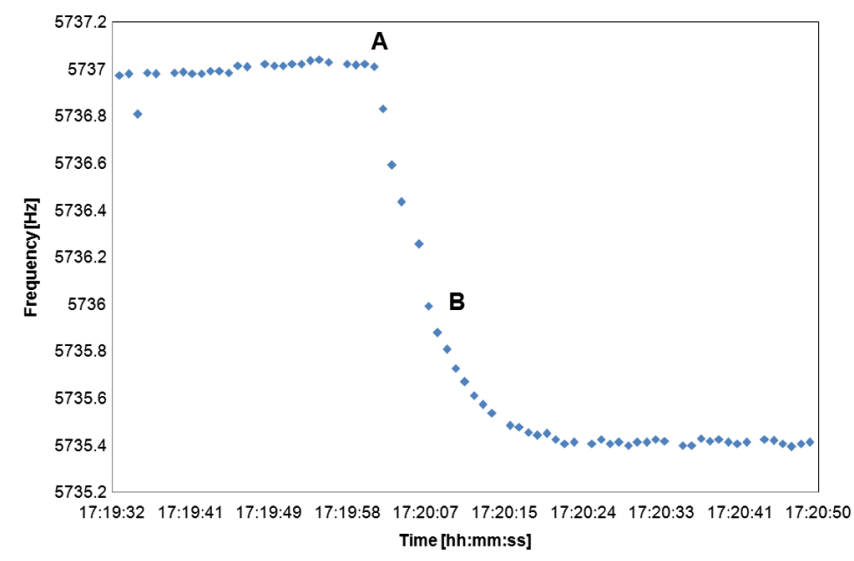

FIG. 7. Plot of frequency vs time for response time measurement. Point A: laser on; point B: temperature increase reaches $63.2 \%$ of saturation.

a $100 \mu \mathrm{m}$ diameter tungsten wire with a graphen layer of thickness $5 \mu \mathrm{m}$ is expected to decrease the response time of the monitor by half.

The response time of the LA-VWM was also found experimentally. The main view of the experimental setup is presented in Fig. 6. The LA-VWM device was mounted on the vacuum port, and a diode laser was used to irradiate the target wire.

The laser light was focused on the target wire center and periodically switched on and off. When the laser was on, we observed the frequency change shown in Fig. 7. The value of the response time measured from this experiment was approximately $6.7 \mathrm{~s}$, which is in good agreement with the analytic estimation for tungsten wire in vacuum (see Table I).

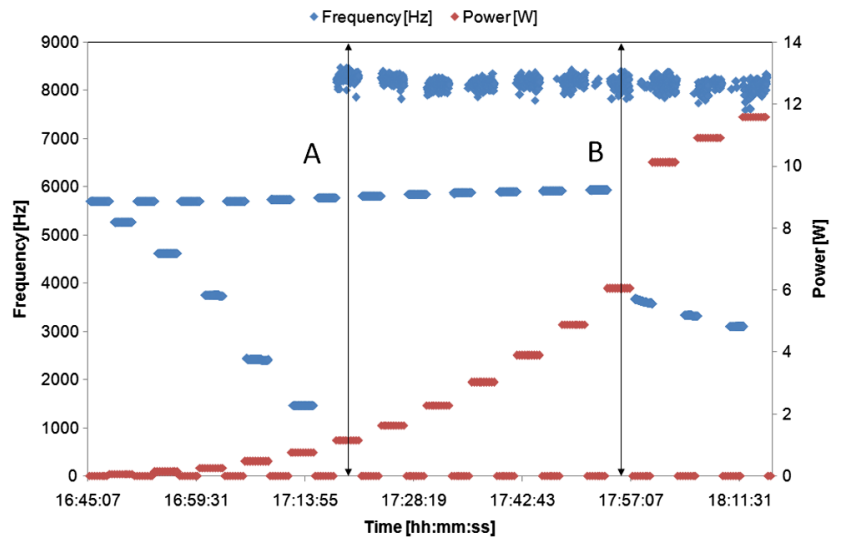

FIG. 8. Frequency change during target wire heating by a dc current. Blue dots: vibrating wire frequency; red dots: power dissipated into the target wire. After the power level of $1.15 \mathrm{~W}$ (indicated by line A), unstable parasitic frequencies $(\sim 8000 \mathrm{~Hz})$ appeared. When the current was switched off, stable oscillation of the vibrating wire was recovered. These processes continued until the power deposition reached $6.04 \mathrm{~W}$ (indicated by line B); thereafter the mechanical properties of the wire were irreversibly changed.

\section{LA-VWM SENSOR THERMAL CHARACTERISTICS}

In the previous experiment using a laser, we could observe only the dynamic heating process without exact information about how much power was indeed accepted by the wire. To investigate the dependence of the vibrating wire frequency on the power deposited into the target wire, we carried out another experiment in which the wire heating was produced by a dc current flowing through the wire. Measurements of the current and voltage on the wire provide exact information on how much power is dissipated into the wire.

The monitor was placed in a vacuum bell glass and pumped by a turbo molecular pump down to about $10^{-4}$ mbar. The temperature of the LA-VWM unmovable

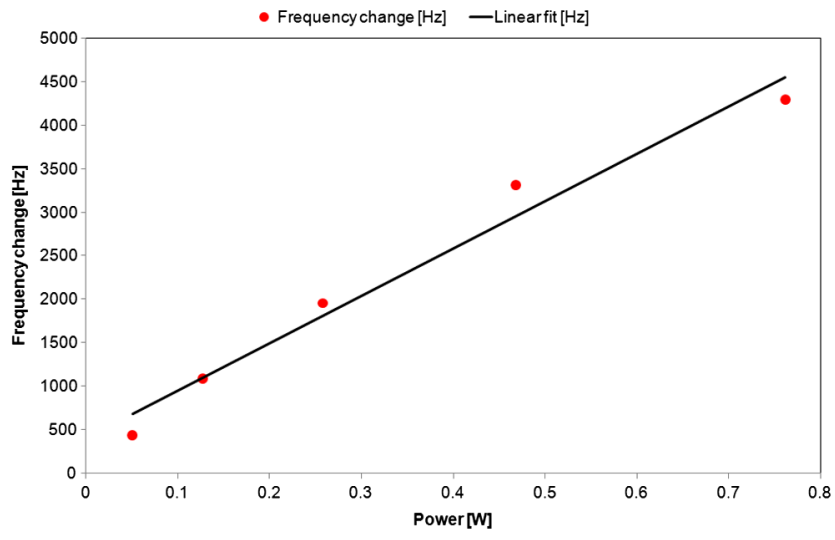

FIG. 9. LA-VWM frequency dependence on the power deposition in the target wire by a dc current. 


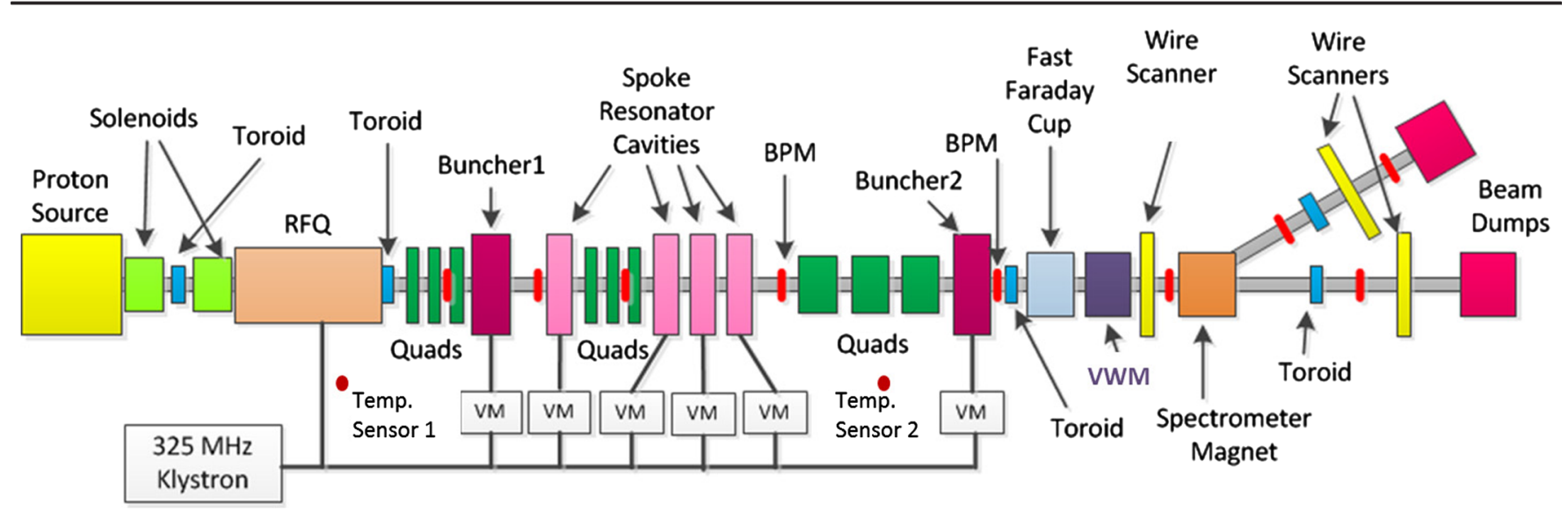

FIG. 10. Block diagram of the HINS beam line configuration used for the beam test of the LA-VWM. The ambient temperature is monitored by the two temperature sensors shown in red circles.

arm was monitored by a platinum thermometer. The target wire was heated by a dc current for $3 \mathrm{~min}$ and then switched off for another $3 \mathrm{~min}$. This process was repeated while increasing the current slowly at each step (see Fig. 8).

For stable wire oscillation generation by an electronic circuit, it is required that the wire should be stressed at some level. When the wire tension is too low, the oscillation generation becomes unstable. We observed such an unstable behavior at a power level of $0.76 \mathrm{~W}$. As the deposited power increased further $(>1.15 \mathrm{~W})$, the wire became much less taut and unstable frequencies of about $8000 \mathrm{~Hz}$ appeared (see line A in Fig. 8). These parasitic frequencies seemed to be produced by the resonances in the electronic gain circuit with a low $Q$ factor. When the power dissipation reached about $1.6 \mathrm{~W}$, the wire became red hot. When the current was turned off, however, stable generation of the wire oscillations could be recovered. On the other hand, if the deposited power increased beyond $6.04 \mathrm{~W}$ (see line B in Fig. 8), irreversible changes in the mechanical properties of the wire occurred, and stable wire oscillation generation became impossible.

Experimental results of the frequency dependence on the power during the initial phase of the current increase are shown in Fig. 9. The slope of a linear fit to the data is about $8.5 \mathrm{~Hz} / \mathrm{mW}$. We note that the minimum detectable frequency shift of about $0.01 \mathrm{~Hz}$ corresponds to the power deposition of $1 \mu \mathrm{W}$.

Although the homogeneous distribution of power deposition along the wire differs from the actual beam power deposition (which is mainly focused in the wire center), this experiment provided approximate characteristics of the LA-VWM. In Ref. [8] we have developed a visual basic program to calculate the temperature profile along the wire as a result of distributed thermal flux. The mean value of the temperature profile determines the final tension of the wire and the frequency response of the VWM. Estimations based on this program indicate that the mean value of the temperature for a Gaussian beam $(1 \mathrm{~mm}$ rms beam radius) is $\sim 40 \%$ higher than the case of uniform power deposition.

\section{BEAM MEASUREMENTS AT THE HIGH INTENSITY NEUTRINO SOURCE FACILITY OF FERMILAB}

The LA-VWM with an aperture size of $60 \mathrm{~mm}$ was installed at the Fermilab High Intensity Neutrino Source facility [13-14] to test its performance for actual transverse beam profile measurements. The HINS front end is composed of a $50 \mathrm{keV}$ proton source, a low energy beam transport line with solenoidal focusing, and a $2.5 \mathrm{MeV}$ radio frequency quadrupole (RFQ). Figure 10 shows a block diagram of the HINS beam line with various beam instrumentation, which includes Beam Position Monitors, toroids, and wire scanners. The spoke cavities and buncher cavities were not used for this test. The typical beam parameters for this measurement are summarized in Table II. Note that the RFQ was operated without water cooling; therefore the repetition rate of the beam pulse was limited to $1 \mathrm{~Hz}$. The LA-VWM (shown in the purple box in

TABLE II. HINS beam parameters.

\begin{tabular}{lcc}
\hline \hline Beam Parameters & Value & Unit \\
\hline Energy & 2.5 & $\mathrm{MeV}$ \\
\hline Species & Proton & $\cdots$ \\
\hline Nominal bunch frequency & 325 & $\mathrm{MHz}$ \\
\hline Nominal bunch spacing & 3.1 & $\mathrm{~ns}$ \\
\hline Pulse length & $80-190$ & $\mu \mathrm{s}$ \\
\hline Pulse repetition rate & 1 & $\mathrm{~Hz}$ \\
\hline Average beam current & $0.01-2.8$ & $\mathrm{~mA}$ \\
\hline \hline
\end{tabular}



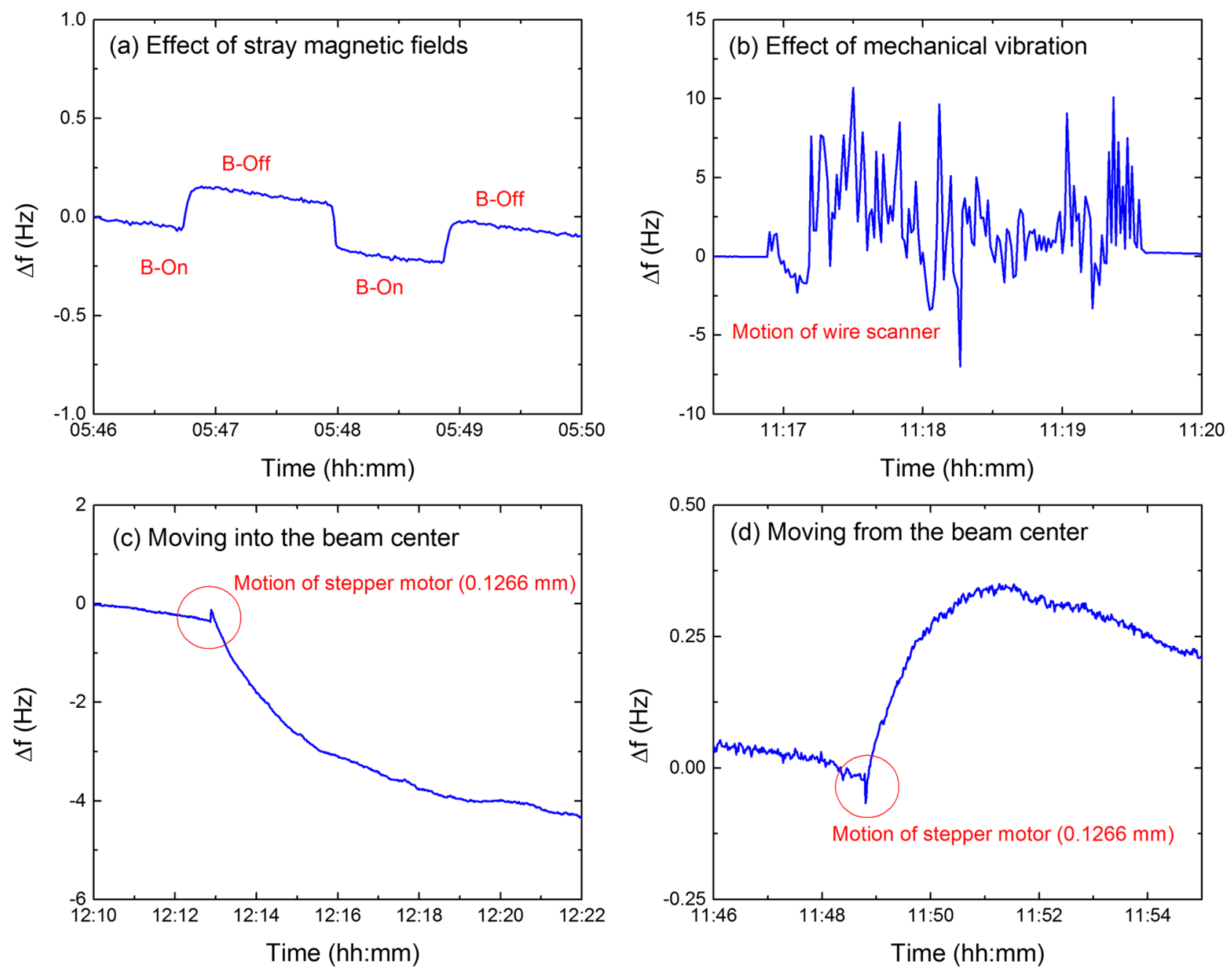

FIG. 11. Characteristics of the LA-VWM during the beam test at the HINS facility.

Fig. 10) was installed next to the first wire scanner that was located in front of the spectrometer magnet. The LA-VWM was moved into the beam center by a stepper motor controlled translational stage ( $0.1266 \mathrm{~mm}$ step size). For the initial test, we used 0.1-mm-thick stainless steel for both the target and vibrating wires.

During the beam test, we observed several notable characteristics of the LA-VWM. Figure 11(a) indicates that stray magnetic fields around the LA-VWM can shift the measured frequency abruptly. This effect can be explained by the change of the field distribution in the monitor's magnetic field generation system. Here, we turned on and off the spectrometer magnet which is located about $0.5 \mathrm{~m}$ downstream of the LA-VWM. In Fig. 11(b), we investigated the effect of mechanical vibration. When the wire scanner next to the LA-VWM was moved by stepper motor, the mechanical vibrations seemed to be transferred along the beam pipe and vacuum chamber and induced noise in the frequency measurements. Hence, for the rest of the experiments, we operated the wire scanner and the LA-VWM separately. Figures 11(c) and 11(d) show

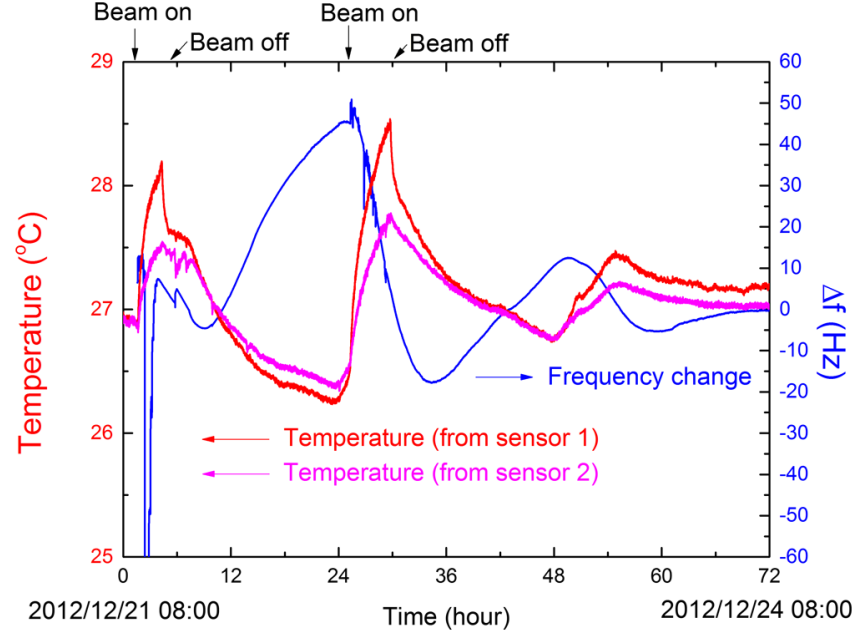

FIG. 12. Effects of the ambient temperature on the frequency change. The frequency change of the vibrating wire and the temperature of the HINS facility were monitored over three full days. On the first two days, the beam was turned on, while on the third day, there was no experiment. The position scanning of the LAWVM was done only for the first day. On the second day, the LAWVM was parked in the outermost position from the beam center. 
the typical frequency responses of the LA-VWM to the proton beam. When the LA-VWM was moving toward the beam center where the current density was high, the vibrating wire frequency went down. On the other hand, when the LA-VWM was moving away from the beam center to the low-density region, the wire frequency went up. Since we used stainless steel for the target wire, the response time is rather long ( $2 \mathrm{~min})$ as estimated in Table I. As shown in the red circles in Figs. 11(c) and 11(d), there are sudden jumps in the frequency readings, which are likely due to the motion of the stepper motor when changing the position of the LA-VWM. These frequency jumps are relatively small and thus do not affect our measurements significantly. The stepper motor stayed off during the frequency measurement for the given scanning position, so no further mechanical or electromagnetic noise was involved. It is interesting to note that in both Figs. 11(c) and 11(d), the frequencies are slowly decreasing before and after the wire positions are changed.
To investigate the cause of this slow frequency drift, we measured the ambient temperature of the HINS facility and compared it with the frequency drift. As illustrated in Fig. 12, we found a strong correlation between the ambient temperature change and the frequency drift. The ambient temperature was monitored by two temperature sensors (see Fig. 10) over three days. One was placed near the RFQ, and the other was located at the last quadrupole triplet. On the first two days, the beam was on and we observed sharp increases in the ambient temperature which seemed to result in the slow frequency decrease of the LA-VWM during the experiments. When the beam was on, a lot of heat was generated from several beam line components. We figured out that the two normal-conducting solenoidal magnets in the low energy beam transport were the major heat sources. Even when the beam was off, the ambient temperature still drifted due to the temperature swing inside the HINS building. This frequency dependence on the ambient
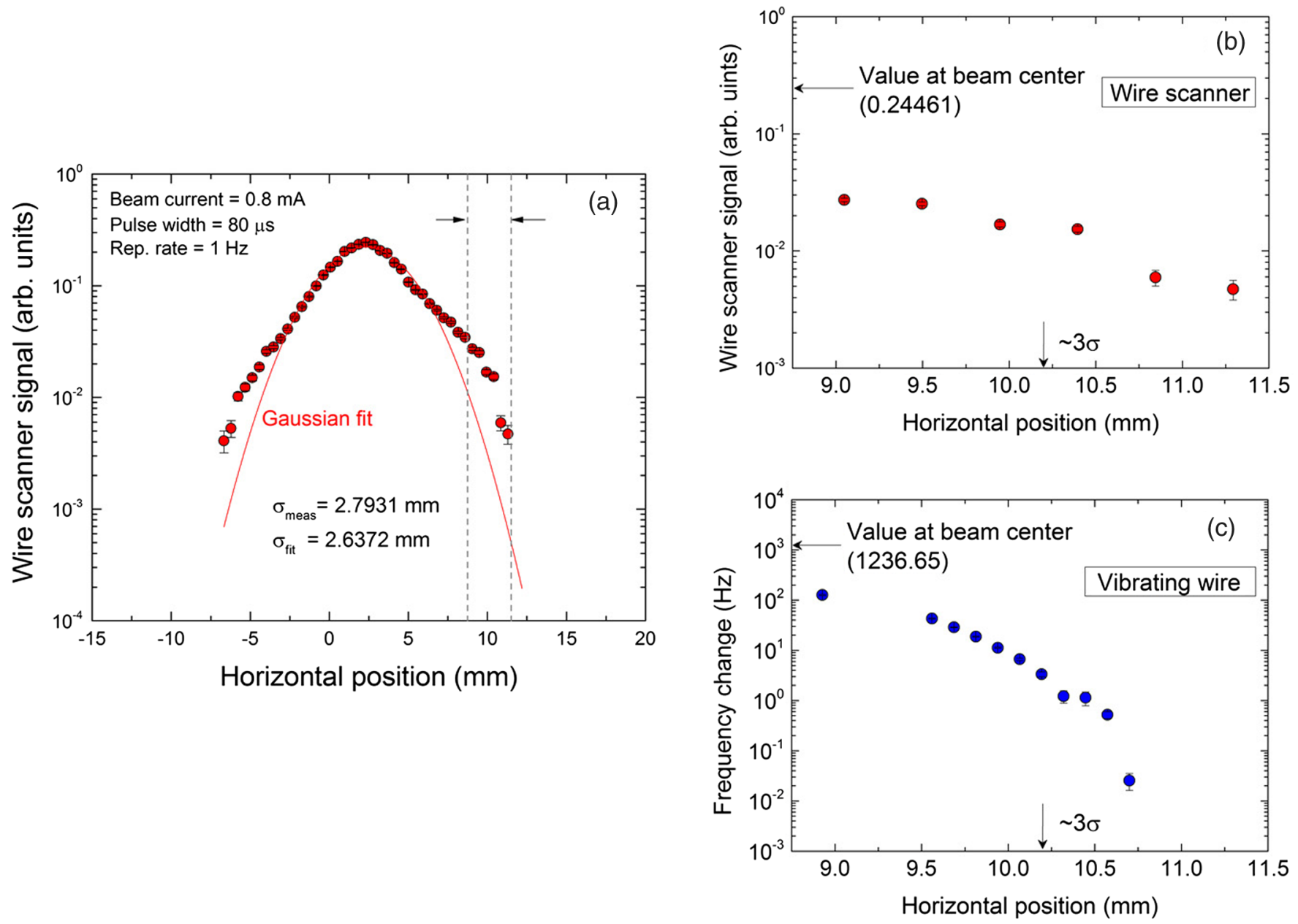

FIG. 13. A reference beam profile is measured by the wire scanner in the horizontal direction (a), and the comparisons between the wire scanner (b) and the vibrating wire (c) are made in the low-density tail region [indicated by dashed lines in (a)]. In (b) and (c), the signal values at the beam center are indicated by the horizontal arrows. 
temperature is consistent with our bench test result in Fig. 4. Note that the sudden drop of the LA-VWM frequency on the first day (see the initial part of the caption of Fig. 12) was due to the position scanning. For the rest of the measurements, we calculated the frequency change after compensating for the effect of the temperature drift. Therefore, for accurate measurements of the beam halo, it would be important to control the ambient temperature around the vibrating wire assembly.

To have a reference profile, we measured the horizontal beam profile using the wire scanner which was located next to the LA-VWM. The wire scanner had a 0.125 -mm-thick molybdenum wire, and the induced currents in the wire were converted into a voltage output via a I-V conversion circuit. Since the total beam current was known from the beam toroid, we could calibrate the wire scanner signal into the line current density by making use of a proportionality factor. The peak in the wire scanner signal corresponded to $\sim 0.121 \mathrm{~mA} / \mathrm{mm}$. As the beam current $(\sim 0.8 \mathrm{~mA})$ and the repetition rate $(1 \mathrm{~Hz})$ were low for the HINS operation, this peak line current density was equivalent to only $\sim 9.7 \mathrm{nA} / \mathrm{mm}$ for a continuous wave beam. As shown in Fig. 13(a), the beam profile reveals a tail structure (deviation from the Gaussian core) around $3 \sigma$ of the rms beam size. Characteristics of the wire scanner and the vibrating wire are compared in this region. Note that the length of the detection region of the LA-VWM is $60 \mathrm{~mm}$ (much wider than the beam size), so the large amplitude halo particles will not be intercepted by the structural material in this case. As demonstrated in Figs. 13(b) and 13(c), the vibrating wire shows a large dynamic range of $\sim 10^{5}$, while the wire scanner has only $\sim 10^{2}$ for this lowcurrent and low-repetition-rate beam operation. Here, the dynamic range is defined as the ratio between the largest and smallest possible values of the measured signals. Therefore, the LA-VWM has great potential for resolving the detailed structure of the low-density halo region (i.e., $<10^{-4}$ of the beam core density [1]).

Fermilab is planning to build a multi-MW proton accelerator facility called Project-X $[12,13]$, which will support a world-leading intensity frontier particle physics program. The initial stage of Project- $X$ will be based on a continuous wave superconducting linac. To minimize the technical risks, particularly in the front end of Project-X, a research and development program called the Project-X Injector Experiment (PXIE) has been proposed [13]. One of the challenges is to characterize and control the beam halo formation because even a small amount of beam loss would seriously limit the performance of the superconducting rf cavities. Therefore, the beam halo measurement is one of the critical research and development items, and further investigation of the vibrating wire is being considered.

\section{CONCLUSION}

A new and more compact modification of the VWM with separate target and vibrating wires was developed. The new monitor has a much larger aperture size than the conventional VWM, and thus enables us to measure transverse beam halos more effectively.

Dependencies of the vibrating wire frequency on the target and ambient temperatures were investigated. Estimations of the LA-VWM response time were made for different parameters sets, which allowed us to choose the proper materials for the LA-VWM. Measurements of the target wire response time were also made by means of laser irradiation, which demonstrated that our theoretical estimations of the response time were valid.

To define the range of the acceptable thermal loads on the LA-VWM target wire, a set of experiments were carried out. Target wire heating by the beam particles was simulated by a dc current, and the maximum limit of the power deposition was obtained. Below the power limit, the wire could have been repeatedly exposed to heat without any irreversible changes in the wire material. When the wire was heated to approximately $1500^{\circ} \mathrm{C}$ (for the case of a tungsten wire), it became incandescent, and we found that the stable generation of wire oscillation was impossible afterward.

All the data presented here, including the beam test results at the HINS facility, indicate that the LA-VWM could be a useful diagnostic instrument in many present and future accelerators and particularly effective for the transverse beam halo measurements.

\section{ACKNOWLEDGMENTS}

The authors are very thankful to J. Bergoz, S. Artinian, $\mathrm{H}$. Bayle, and S. Paul for many useful discussions. Work is partly supported by the Yerevan Physics Institute. The authors would like to thank B. Hanna and J. Steimel for their help in operating the HINS facility. This work is partly supported by the U.S. Department of Energy (No. DEAC02-07CH11359) and Handong Global University (Pohang, Korea) Research Grants 2011.

[1] K. Wittenburg, CERN Accelerator School 2009-005, p. 557 (2009).

[2] K. Wittenburg, Proceedings of the 39th ICFA Advanced Beam Dynamics Workshop, High Intensity High Brightness Hadron Beams, HB'2006, Tsukuba, Japan, 2006 (KEK, Tsukuba, Japan, 2006), p. 54.

[3] J. Egberts and C. P. Welsch, JINST 5, P04010 (2010).

[4] H. D. Zhang, R. B. Fiorito, A. G. Shkvarunets, R. A. Kishek, and C. P. Welsch, Phys. Rev. ST Accel. Beams 15, 072803 (2012).

[5] S. G. Arutunian, N. M. Dobrovolski, M. R. Mailian, I. G. Sinenko, and I. E. Vasiniuk, in Proceedings of the 18th 
Particle Accelerator Conference, New York, 1999 (IEEE, New York, 1999), p. 2105.

[6] S. G. Arutunian, N. M. Dobrovolski, M. R. Mailian, I. G. Sinenko, and I. E. Vasiniuk, Phys. Rev. ST Accel. Beams 2, 122801 (1999).

[7] S. G. Arutunian, N. M. Dobrovolski, M. R. Mailian, and I. E. Vasiniuk, Phys. Rev. STAccel. Beams 6, 042801 (2003).

[8] S. G. Arutunian, M. R. Mailian, and K. Wittenburg, Nucl. Instrum. Methods Phys. Res., Sect. A 572, 1022 (2007).

[9] S. G. Arutunian, Proceedings of Beam Instrumentation Workshop, BIW'08, Lake Tahoe, MI 2008 (LBNL, Berkeley, CA, 2008), pp. 1-7.

[10] S. G. Arutunian, K. G. Bakshetyan, N. M. Dobrovolski, M. R. Mayilyan, V. A. Oganessian, H. E. Soghoyan, I. E. Vasiniuk, and K. Wittenburg, in Proceedings of the 9th European Particle Accelerator Conference, EPAC-2004, Lucerne, Switzerland (EPS-AG, Lucerne, 2004), pp. 24572459.

[11] S. G. Arutunian, A. E. Avetisyan, N. M. Dobrovolski, M. R. Mailian, I. E. Vasiniuk, K. Wittenburg, and R. Reetz, in Proceedings of the 8th European Particle Accelerator Conference, Paris, 2002 (EPS-IGA and CERN, Geneva, 2002), pp. 1837-1839.
[12] Project-X Team, Fermilab, Project-X Reference Design Report No. Project X-doc-776-v5, 2013.

[13] V. E. Scarpine, B. M. Hanna, V. Lebedev, L. Prost, A. V. Shemyakin, J. Steimel, and M. Wendt, in Proceedings of the 3rd International Particle Accelerator Conference, New Orleans, LA, 2012 (IEEE, Piscataway, NJ, 2012), pp. 954-956.

[14] V. Scarpine, B. Webber, J. Steimel, S. Chaurize, B. Hanna, S. Hays, D. Wildman, D. Zhang, and M. Wendt, in Proceedings of the 25th International Linear Accelerator Conference, LINAC-2010, Tsukuba, Japan (KEK, Tsukuba, Japan, 2010), pp. 755-757.

[15] J. Steimel, V. Scarpine, R. Webber, and M. Wendt, in Proceedings of BIW'10, Santa Fe, NM, 2010 (LANL, SantaFe, 2010), pp. 442-445.

[16] S. G. Arutunian, A. E. Avetisyan, M. M. Davtyan, and I. E. Vasiniuk, J. Contemp Phys. 46, 247 (2011).

[17] Time constant: http://en.wikipedia.org/wiki/Time_ constant.

[18] A. K. Geim, K. S. Novoselov, Nat. Mater. 6, 183 (2007).

[19] http://www.graphenetechnologies.com/.

[20] http://www.graphenea.com/. 